\title{
Vitrification, an efficient cryopreservation procedure but still rising some debates
}

\author{
Pierre Vanderzwalmen ${ }^{1,2,3}$ \\ Fabien Ectors ${ }^{4}$ \\ Astrid Stecher ${ }^{1}$ \\ Delphine Connan ${ }^{3}$ \\ Deborah Desmet ${ }^{2}$ \\ Jacqueline Greindl ${ }^{2}$ \\ Nicolas Zech ${ }^{1}$ \\ Barbara Wirleitner ${ }^{1}$
}

${ }^{1}$ IVF Centers Prof. Zech, Bregenz, Austria

${ }^{2}$ IVF Unit, Centre Hospitalier Inter-regional Edith Cavell (CHIREC), Braine 1 Alleud - Bruxelles, Belgium

${ }^{3}$ GIGA \& FARAH Research, Centers, ULg, Liège, Belgium

${ }^{4}$ GIGA \& FARAH Transgenic Platform, ULg, Liège, Belgium

Address for correspondence:

Pierre Vanderzwalmen

Av du bois de chapelle 4

B 1380 Lasne, Belgium

E-mail: pierrevdz@hotmail.com

\section{Summary}

Since the implementation of efficient vitrification techniques, the percentage of cryopreservation cycles worldwide increased dramatically. Moreover, there is a trend toward "vitrification-all strategy" after IVF with single blastocyst transfer in a subsequent warmed cycle.

With the final objective of increasing the cumulative pregnancy rate, efficient, standardized, reliable, harmless, aseptic vitrification is mandatory. Vitrification procedure consists of several steps. Some of them generate debates.

In this review several phases of the procedure that aroused debates are discussed. For example: (1) which blastocysts to select before and after vitrification? (2) is it required to collapse the blastocoele? (3) is scepticisms about the use of high concentrations of cryoprotectants justified? (4) is it possible to vitrify in reduce cooling conditions and achieve aseptic vitrification? (5) is the warming process more important than the cooling one, (6) and finally concerns about the stability of vitrified biological materials over time.

KEY WORDS: aseptic vitrification, cryoprotectant concentration, open closed carrier, cooling-warming rates.

During the last 15 years, due to a remarkable improvement in the efficiency of vitrification techniques as alternative to the classical slow freezing procedure, the proportion of births following transfer of cryopreserved embryos has increased dramatically (1-9). With the policy of single blastocyst transfer, an increasing proportion of supernumerary blastocysts are vitrified either on day 5 or 6 (6).

Currently there is a trend toward cryopreserved all strategy after IVF with transfer of a warmed embryo in a subsequent cycle (10). There have been reports of greater implantation and pregnancy rates with frozen embryo transfer than with fresh autologous embryo transfer, suggesting superior endometrial receptivity in the absence of ovarian stimulation $(11,12)$. Moreover, blastocysts vitrification is a valuable option when they originated from in vitro maturation cycles $(3,13)$.

Also, the rationale is that transfer of an embryo into a more "physiologic environment" would result in greater pregnancy rates and potentially a decrease of global both maternal and perinatal morbidity (14).

\section{Principle, description and questions that arise during the different phases of a vitrification procedure}

Vitrification and warming of embryos (blastocysts) consist of several steps (Figure 1):

Step one: selection of blastocysts before vitrification with or without artificial blastocoele collapsing. 


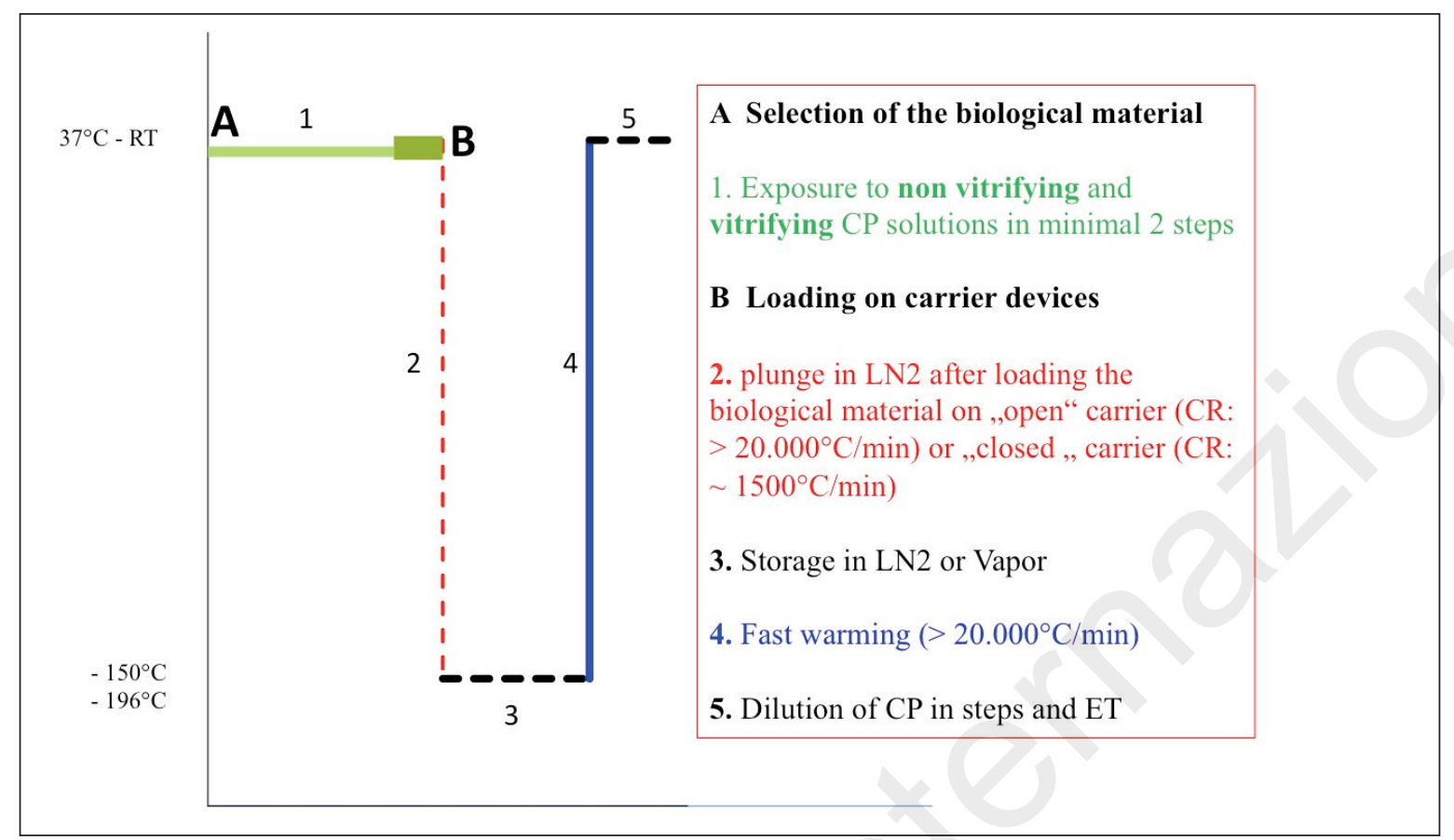

Figure 1 - Vitrification: steps.

Step two: exposure of blastocysts to the croprotectant (CP) solutions.

Step three: loading on the carrier device and plunging it in liquid nitrogen $\left(\mathrm{LN}_{2}\right)$.

Step four: storage in $\mathrm{LN}_{2}$ containers.

Step five: the warming process.

Step six: dilution of the CP.

Step seven: selection of warmed blastocysts before embryo transfer (ET).

Even though the basic principle to vitrify biological material is quite similar according to different protocols, some questions are still matter of debates.

The aim of this manuscript is to bring our opinions about several interrogations and principally in case of vitrification of blastocysts.

\section{Step one (a): selection of blastocysts before vitrification, should we discard the second quality one?}

A pivotal question is the selection at the blastocyst stage. Which ones should we vitrified and which ones to discard? This raises the question on how to define a good quality blastocysts? The one that looks morphologically normal or the one that implant?

Grading of blastocyst morphology incorporates as- sessment of degree of expansion of the blastocyst and the quality of the inner cell mass and the trophectoderm $(15,16)$. Several studies showed that trophectoderm was determined to be statistically significantly related to the rate of ongoing pregnancy and miscarriage. By contrast, neither inner cell mass nor blastocyst expansion was statistically significantly related $(17,18)$. In a more recent article, Ahlström et al. (19) analyse which prefreeze morphological parameters can be used to predict live birth outcomes after vitrified/warmed blastocyst transfer cycles. They stated that blastocoele expansion and trophectoderm grade were identified as the most significant pre-freeze morphological predictors of live birth.

According to our experience, we suggest that in principle one might be confronted by mainly four groups on day 5 (Figure 2).

The good ones with an Inner Cell Mass (ICM) and trophectoderm (TE) classified as A and /or B accompanied with a degree of expansion 5, 4, 3 and 2 according to the morphological scoring criteria of Gardner et al. (20).

The second group is composed of embryos with an ICM and TE classified as B and C with a degree of expansion from expansion 2 to expanded blastocyst.

The third encompasses early blastocysts of good and non top quality.

The fourth group includes those with a delay of de- 


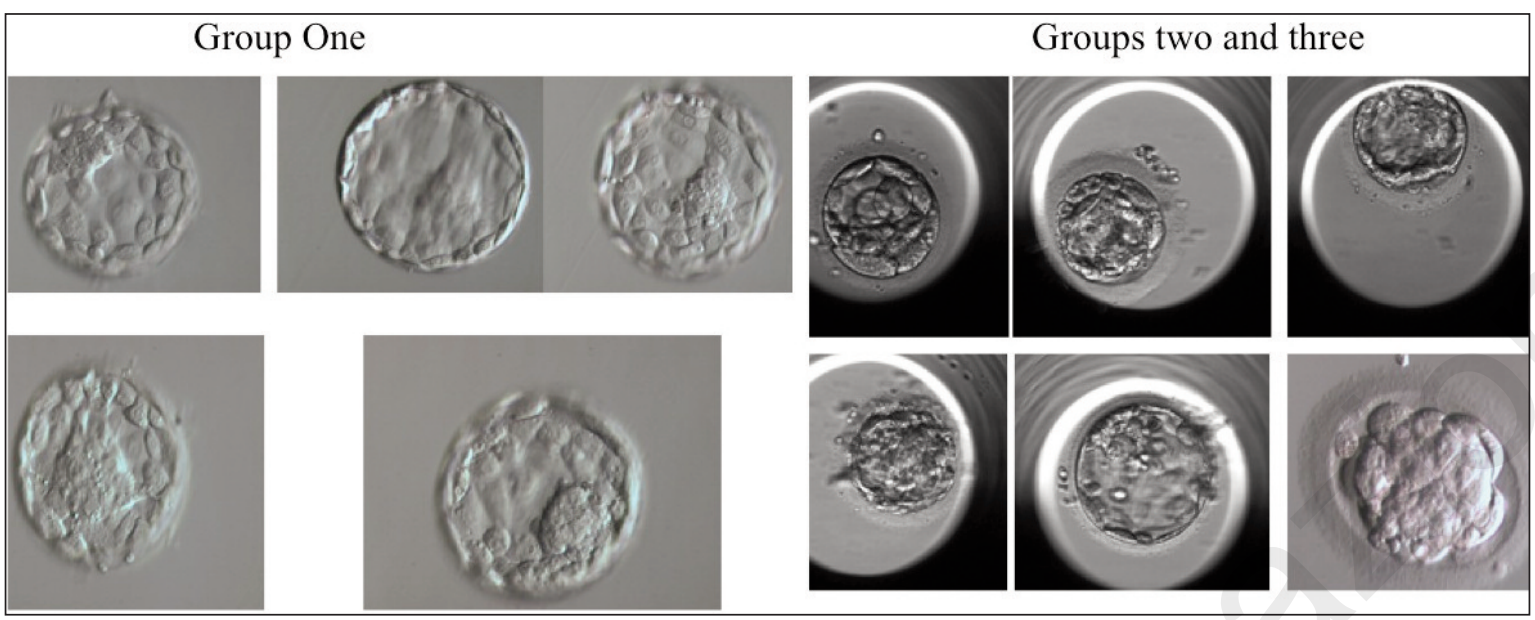

Figure 2 - Selection of blastocysts before vitrification: should we discard the second quality one?

velopment and revealing no sign of cavitation and those that show i.e. an arrested development on day 3 . In this group, culture to day 6 after or before vitrification is performed.

Interestingly, according to the data which is and was recently presented in several congresses worldwide, only blastocysts which fulfill the criteria of the first group are selected for vitrification and more rarely embryos from the second one. Those which encompass groups III and IV are mostly not selected. Thus, results always appear outstanding at a first glance. However, if all the patients who had no ET and those, where no surplus embryos were cryopreserved due to poor mor- phology would be included in the data the overall success rates would look rather disappointing. But, do we have to present only excellent results, knowing that if we look the patient point of view, their advices would be totally different?

According to our experience (Tables 1 and 2), we still observed birth rates around $25 \%$ when lower quality blastocysts were transferred. For those from the fourth group, an extra culture period to day 6 , is a valuable option instead of discarding them according to tangible criteria. We observe higher birth rate after vitrification of bad quality embryo on day 5 or 6 as compared to fresh embryo transfers of similar quality.

Table 1 - Birth rates after fresh blastocysts transfers and aseptic vitrified blastocysts transfers.

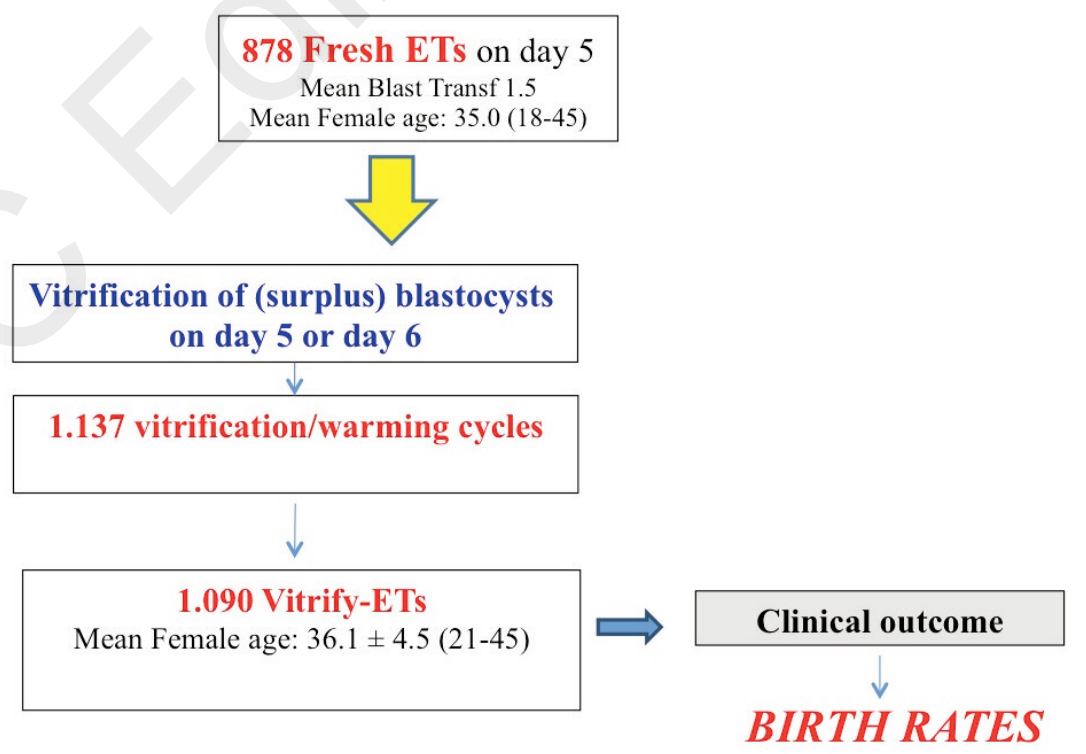

Data from Zech IVF center, Bregenz 
Table 2 - Survival rates after vitrification/warming.

\begin{tabular}{|c|c|c|c|c|c|}
\hline $\begin{array}{l}\text { Blastocyst quality before } \\
\text { vitrification }\end{array}$ & Cycles & $\begin{array}{l}\text { Age of } \\
\text { patients at } \\
\text { vitrification }\end{array}$ & $\begin{array}{l}\text { Age of } \\
\text { patients at V- } \\
\text { ET }\end{array}$ & $\begin{array}{l}\text { Survival } \\
\text { rates }\end{array}$ & $\begin{array}{c}\text { Birth } \\
\text { rate }\end{array}$ \\
\hline \multirow{3}{*}{ 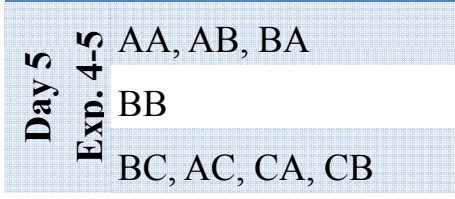 } & 311 & 34.2 & 35.5 & $87 \%$ & $30 \%$ \\
\hline & 237 & 34.8 & 36.1 & $85 \%$ & $32 \%$ \\
\hline & 88 & 34.6 & 35.4 & $88 \%$ & $35 \%$ \\
\hline \multirow{3}{*}{$\begin{array}{l}\text { ì } \mathrm{AA}, \mathrm{AB}, \mathrm{BA} \\
\stackrel{\mathrm{i}}{\mathrm{B} B} \\
\mathrm{BC}, \mathrm{AC}, \mathrm{CA}, \mathrm{CB}\end{array}$} & 125 & 35.2 & 35.8 & $89 \%$ & $29 \%$ \\
\hline & 203 & 35.6 & 36.7 & $90 \%$ & $31 \%$ \\
\hline & 66 & 35.1 & 36.2 & $92 \%$ & $23 \%$ \\
\hline \multirow{3}{*}{ 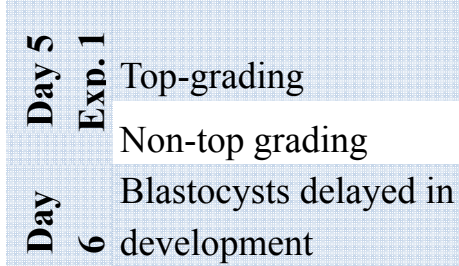 } & 44 & 36.7 & 38.7 & $86 \%$ & $25 \%$ \\
\hline & 34 & 35.1 & 36.1 & $77 \%$ & $22 \%$ \\
\hline & 29 & 37.3 & 37.8 & $98 \%$ & $37 \%$ \\
\hline
\end{tabular}

Also acceptable results were obtained after vitrification of blastocysts that originated from embryos that were not selected either for fresh ET and cryopreservation on day 3 because of their poor quality. Fifty blastocyst warming cycles resulted in a $76 \%$ survival rate, $44 \%$ clinical pregnancy rate, and $39 \%$ implantation rate (21).

So, is it really an option to reject those blastocysts for transfer or vitrification? May be that some sacrifice these ones in order to beautify their implantation rates?

It is worth mentioning that with the current technology it is difficult to select the right embryos even in a routine IVF lab. Of course when good looking embryos are presented, they will be selected for transfer or for cryopreservation. However, there is still no method or algorithm that will improve the selection. But the more difficult task at present is to select from a cohort of bad looking embryos, one that may implant after fresh ET or cryopreservation. Again, what should we do, keep everything or select?

It should be mandatory to cryopreserve all supernumerary blastocysts of moderate to good quality in order to increase cumulative pregnancy rate. With regard to this, it is very important to achieve a reproducible outcome, especially in terms of survival after warming independently of the quality, to allow high success rates after vitrified/warmed blastocyst transfer.

\section{Step one (b): is it required to collapse the blastocoele?}

With respect to blastocyst quality and survival, the question arises whether artificial shrinkage or collapsing is mandatory (Figure 3)?

Since the size of the blastocoele correspond to the amount of water in this cavity, larger blastocysts might show reduced cryopreservative potential due to ice crystal formation during the cooling step and ice recrystallization (devitrification) during the warming step.

In order to reduce the likelihood of ice crystal formation artificial shrinkage or collapsing of expanded/ blastocysts has been suggested using either micropipettes (1) or laser pulses (22).

However, with the use of vitrification solution containing higher sucrose concentration of $0.75 \mathrm{M}$ instead of $0.5 \mathrm{M}$, we observe that artificial shrinkage of the blastocoelic cavity is not necessary for preventing injury from intracellular ice because of sufficient dehydration during the exposure to the vitrification solution.

In addition, Zech et al. (23) showed the benefit of opening the zona pellucida some hours before the vitrification process. They observed that blastocysts with a larger blastocoelic cavity survived vitrification better when they partially or completely hatched even though short exposure to $\mathrm{CP}$ solu- 


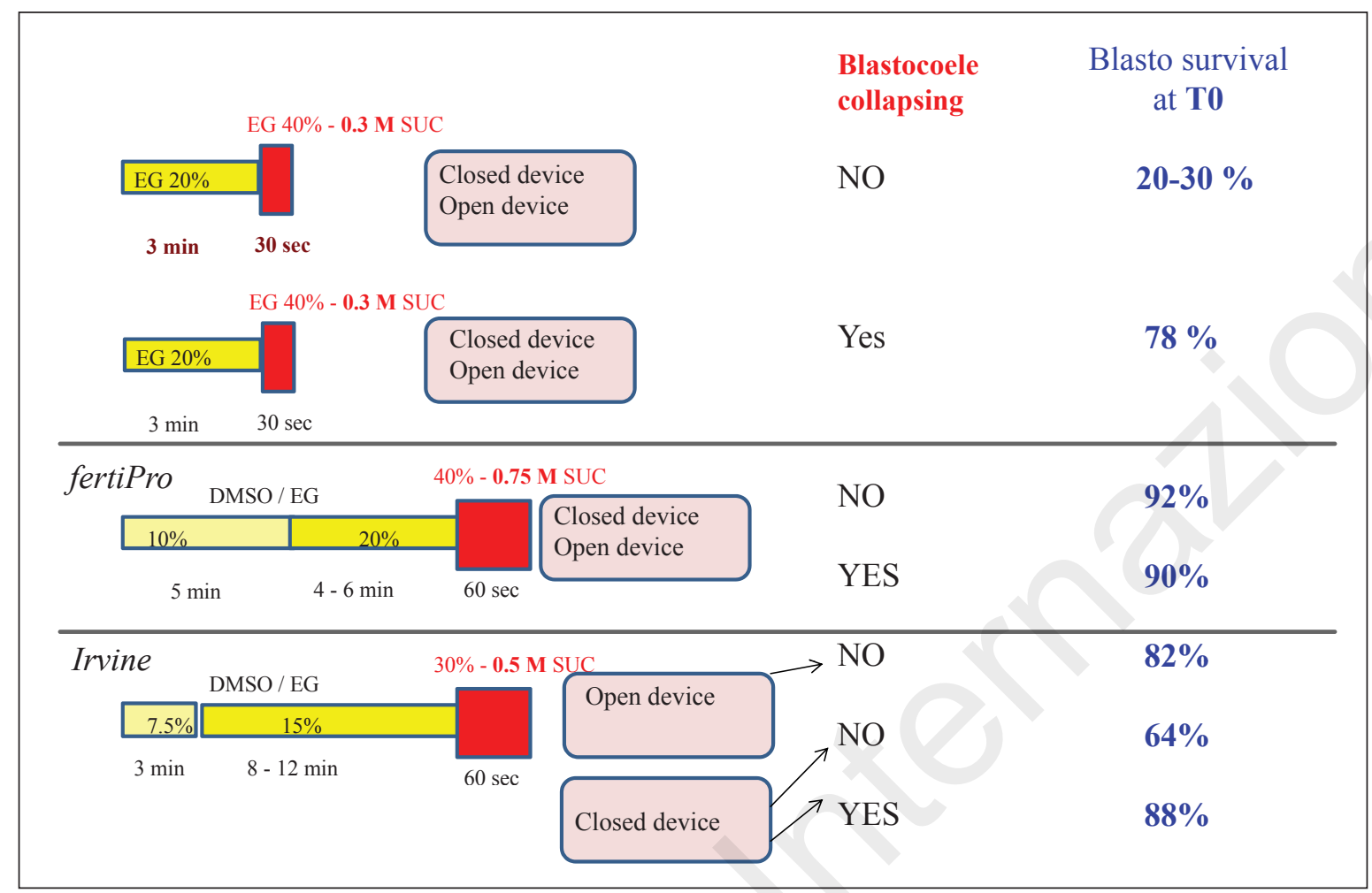

Figure 3 - Blastocoele collapsing: mandatory?

tions. In conclusion, collapsing of the blastocoele is not really necessary and has to be adapted to the rate of dehydration that occurred in the vitrification solution.

\section{Step two: exposure of blastocysts to high concentration of cryoprotectant solutions (CPs). How high the intracel- lular concentration of cryoprotectant (ICCP) truly is?}

\section{Vitrification: conditions to achieve a vitrifica- tion state (Figure 4)}

When the temperature decreases, liquid water can be converted either to a solid crystal or to a solid amorphous glass when the supercooled water is dropped instantaneously below the glass transition temperature. It is solely the skill of being able to prevent ice crystals to form inside the cell (which can happen during the cooling as well during the warming process) that will determine the viability of the embryos. The physical process by which a super viscous liquid solution remain supercooled during the transit through the crystalline phase and reach the solid glassy state when it is cooled below its glass transition temperature $(\mathrm{Tg})$ is called vitri- fication (24). According to this definition, with the application of vitrification, neither in the intra-cellular nor in the extra-cellular spaces, formation of ice crystals is theoretically possible because solidification of a super viscous liquid solution that remains supercooled during the cooling process.

The fundamental issue in all vitrification methods is to achieve and maintain conditions within the cells which guarantee an amorphous state throughout the cooling as well as during the warming process. Independent of the carrier device that determine the cooling and/or the warming rate, the key of success in order to achieve a "glass-like" state depends on an optimal balance between the speed of cooling - rewarming (time and temperature) and the optimal cell dehydration and penetration of $\mathrm{CP}$ when they are exposed to concentrated hypertonic solutions (25).

\section{Principle of exposure to high concentration of CPs}

Before the blastocysts are immersed in $\mathrm{LN}_{2}$, they are exposed to CPs in order to increase the intraand extra- cellular viscosity to a level that the liquid water molecules will solidify so quickly that they will not have time to rearrange themselves into a crystalline structure. To achieve this objective, 


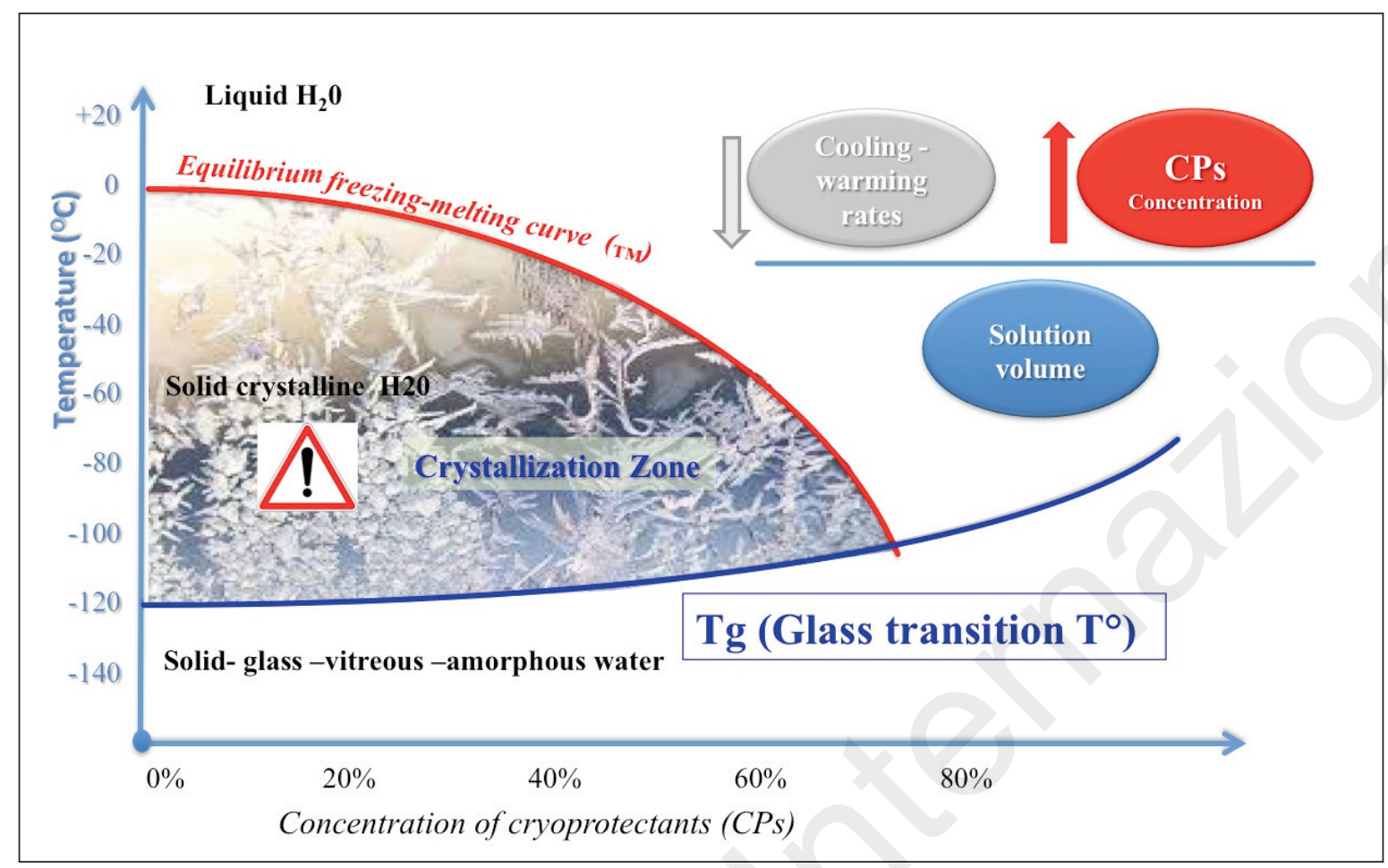

Figure 4 - Parameters that influence the probability of achieving the vitrified state.

in nearly all vitrification methods the blastocysts are exposed to a minimum of two steps of gradually increasing concentrations of non vitrifying solution (nVS) (2.3 M to $3.2 \mathrm{M}$ ) and vitrifying solution (VS) containing penetrating $(4.8 \mathrm{M}$ to $6.4 \mathrm{M})(26$, $30)$ and non-penetrating $(0.5-0.75 \mathrm{M})$ CPs just before being plunged in liquid nitrogen $\left(\mathrm{LN}_{2}\right)$.

The duration of exposure to the permeable CPs is determined by several biophysical factors such as the membrane properties (cellular permeability to water and $\mathrm{CP}$ ), the type and concentration of $\mathrm{CP}$, the surface/volume ratio of the cells, and the rate of cooling and warming (27-29).

\section{Fear about the ICCP reached during exposure to CPS: justified?}

Fear about exposing gametes and embryos to high amounts of CPs that exceed 3 to 4 fold those found in slow freezing (SLF) was the central part of a debate initiated by advocates of the SLF procedure where low concentrations of penetrating CPs are used.

Two investigations were undertaken with mouse zygotes to bring some insights on how high the IC$\mathrm{CP}$ truly is immediately before plunging the blastocysts in $\mathrm{LN}_{2}$ and compared it with the ICCP in a SLF (Figure 5).

With our protocol $(3,11)$, we allow the CPs to penetrate the zygote during the exposure to nVS1
(1.6M) and nVS2 (3.2M), but not until it recovers its initial volume. As consequence, the equilibrium between intra and extracellular CP concentrations is not completely achieved. To reach an intracellular vitrified state, embryos are exposed in the last step to the VS (6.4 M) where sucrose and Ficoll, as non-penetrating $\mathrm{CPs}$, induce dehydration. Thereby various intrinsic macromolecules like (glyco)proteins, salts and also penetrating CPs are concentrated, generating an intracellular environment that enables to reach and maintain a vitreous state during the cooling and warming processes. An extracellular vitrified state is allowed by the high concentrations of CPs in the VS that encapsulates the embryo in a vitrifying sheath.

We have found that the ICCP in mouse zygotes during the vitrification procedure prior to plunging them in liquid nitrogen is approximately equal to $2.14 \mathrm{M}$ and is 3 -fold below the concentration in the VS that coats the cell and even lower than after slow freezing procedure (30) (Figure 6). When incubated in $2.14 \mathrm{M}$ sucrose solution only (D) minimal changes in cell volume occurred. This reduced volume modification indicates that an osmotic quasi-equilibrium exists between intracellular and extracellular compartments. Therefore during VIT, the intracellular osmolarity approaches 2.14 $\mathrm{Osm} / \mathrm{L}$ before the zygotes are plunged in $\mathrm{LN}_{2}$. 


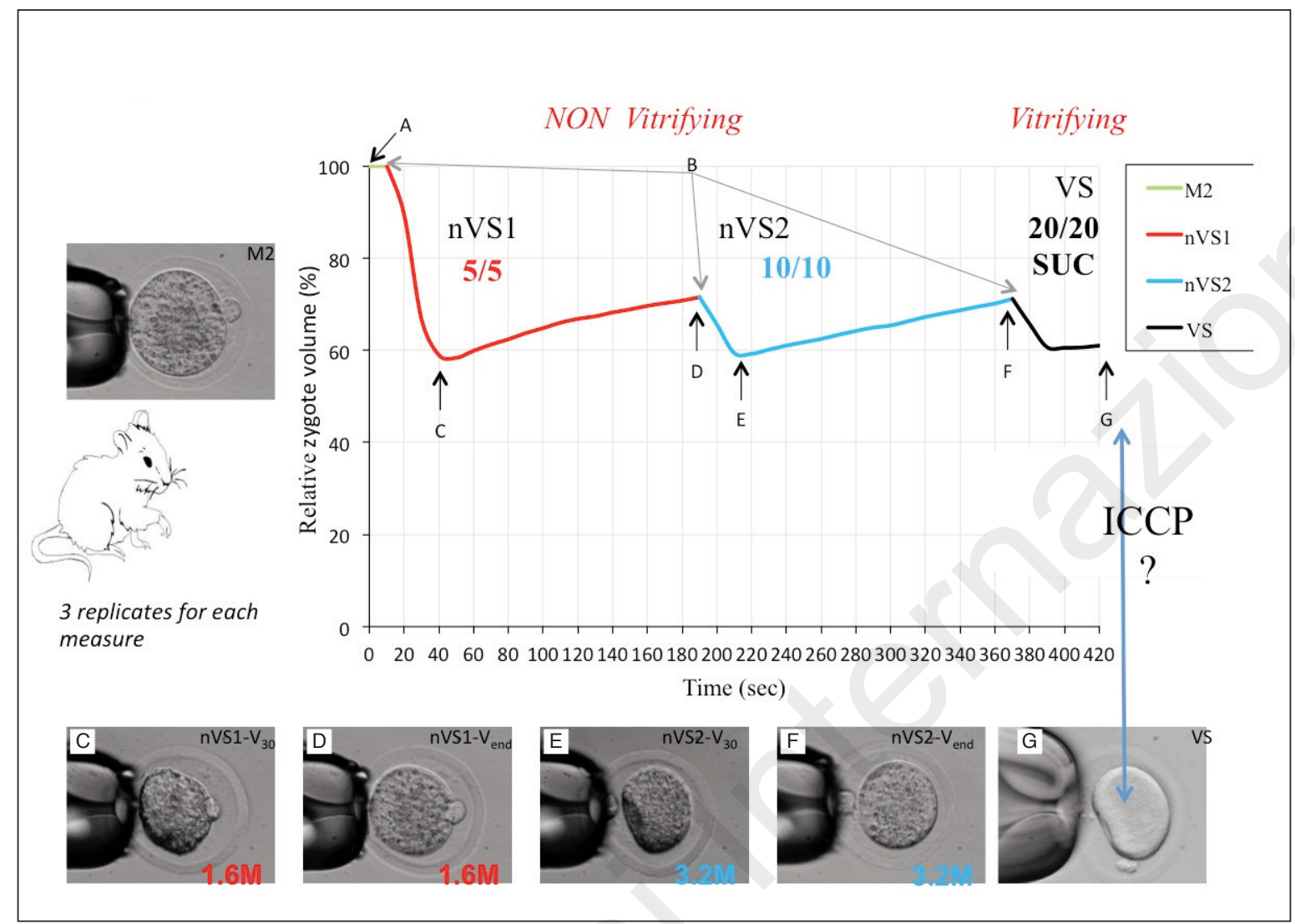

Figure 5 - How high the ICCP truly is immediately before plunging the blastocysts in $\mathrm{LN}_{2}$ ?

We may as consequence state that although slow freezing is the standard cryopreservation method since more than 25 years, few were aware that cell survival is the consequence of the presence of an intracellular vitrified state. This vitrified state is a consequence of a long effect of solution during cooling and is a reflect of a very high concentration of CP. We may therefore suggest that a drop in the survival after SLF is probably more related with osmotic shock after warming than to mechanical injuries due to the formation of intracellular ice crystal. To conclude, we may advice that slow freezing is finally another way to do vitrification.

\section{Step three: loading on a carrier device and plunging in $\mathrm{LN}_{2}$ : can we envisage the end of the 'open' system?}

Open system

It was postulated that ultra-rapid cooling and warming rates (as high as $20.000^{\circ} \mathrm{C}-30.000^{\circ} \mathrm{C} / \mathrm{min}$ ) are mandatory during the vitrification process to reduce the risk of intracellular crystal formation and the concomitant damage to the cell structures (31). To achieve ultra-rapid cooling rates, a very small volume of VS of less than $1 \mu \mathrm{l}$ is deposited on open carrier devices (e.g., Cryotop, Vitriplug, Cryoloop, copper electron microscopy grids), which are directly plunged into $\mathrm{LN}_{2}(1,4,32,33)$.

The advantage of such an approach is that blastocysts are exposed in 2 steps to increasing concentrations of CP. However, only for a short period of time, but long enough to permit the extraction of the intracellular water while limiting the amount of $\mathrm{CP}$ permeating into cells.

One drawback of the "open" carrier devices is that the blastocysts are directly exposed to $\mathrm{LN}_{2}$ during the cooling process as well as during the whole storage time. Although the theoretical risk of cross-contamination, by bacteria, viruses or fungi during cooling or storage in $\mathrm{LN}_{2}$, has been widely debated (34). The potential probability of a toxic effect with reactive chemical compounds present in $\mathrm{LN}_{2}$ raises safety concerns (35).

Various methods for sterilizing $\mathrm{LN}_{2}$ have been proposed or are under development, including ceramic filters (36) or UV-light with subsequent hermetical cryostorage $(37,38)$, or using $\mathrm{LN}_{2}$ vapor for storage (39). Although the probability of an im- 


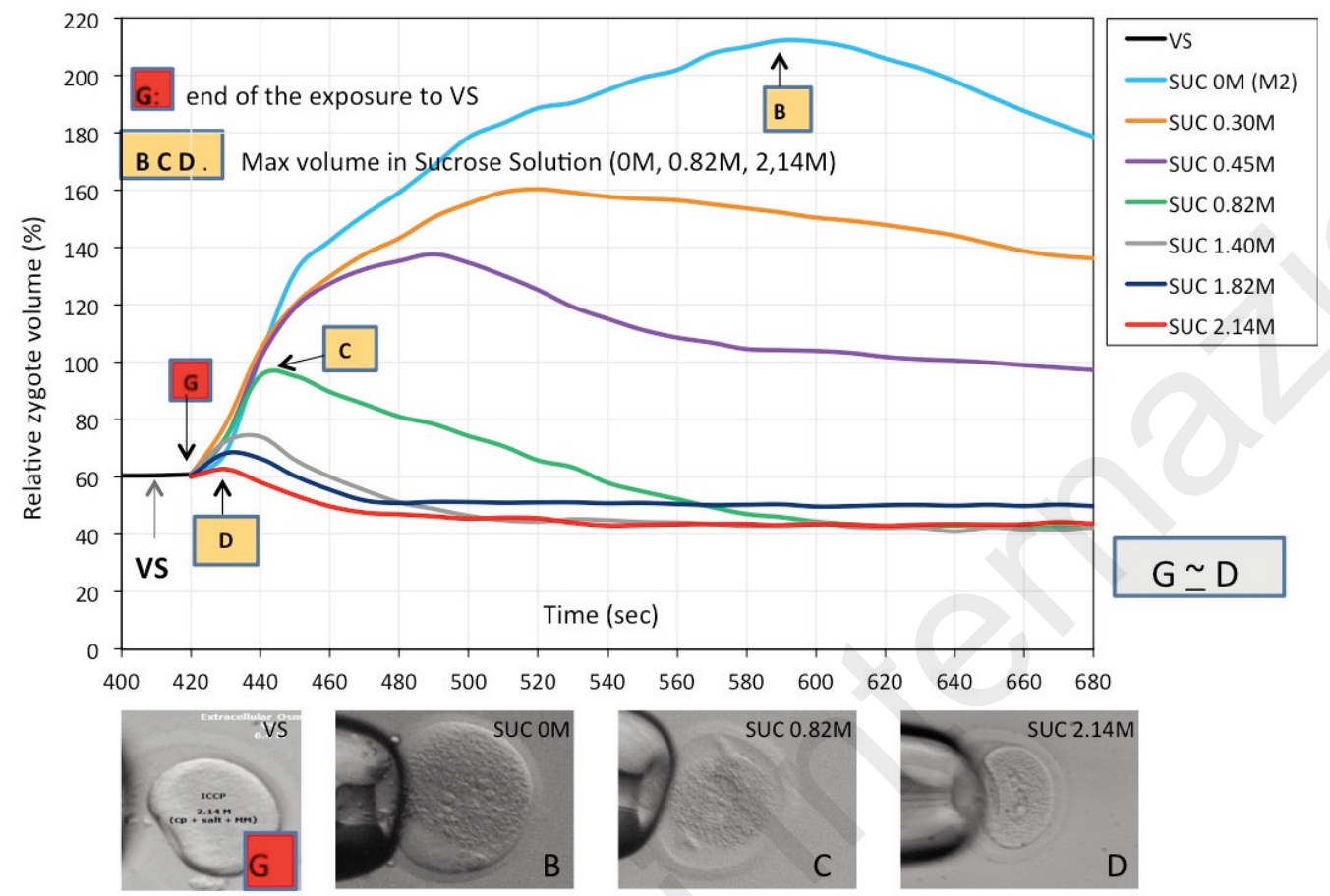

Figure 6 - Evolution of the relative zygote volume when immersed in solutions with various concentrations of SUC following the exposure steps to nVS1, nVS2 and VS.

pairment of cellular structures by contact with $\mathrm{LN}_{2}$ is still being discussed, this risk is important and the ongoing discussion indicates that the storing system, especially in long-term means, should be revised. Even the standard storage conditions and refilling of the tanks pose a hazard when oxygen from surrounding air condenses and mixes with $\mathrm{LN}_{2}$ during the regular opening of the nitrogen tank for routine refilling or whenever straws are added or withdrawn. Although, it is generally assumed that thermally driven reactions do not occur in cells at $-196^{\circ} \mathrm{C}$, it has been reported that in the case of radiation of an $\mathrm{LN}_{2}$ /oxygen mixture a synthesis of oxygen radicals resulting from ozone formation and decomposition cannot be excluded and is even enhanced by the catalytic effect of nitrogen. Mouse oocytes show impaired survival, fertilization rates and embryonic development after prolonged contact with $\mathrm{LN}_{2}$ (40).

\section{Closed system}

The European Directives (2004/23/EC) (41) as well as the FDA directives on tissue and cell storage dictate to adhere to certain safety regulations, ensuring that gametes and embryos are protected from any possible contamination with pathogens and to prevent them from any harmful physical conditions during storage. To achieve the EU directive, a valuable option consists to switch from an open vitrification carrier device to a protocol that entails complete isolation of the biological samples from $\mathrm{LN}_{2}$ during both the cooling process as well as storage by hermetically isolating the embryos from $\mathrm{LN}_{2}$ in the tanks.

A huge difference exists between vitrification protocols with open $\left(>25,000^{\circ} \mathrm{C} / \mathrm{min}\right)$ or closed $\left(<2,000^{\circ} \mathrm{C} / \mathrm{min}\right)$ carrier devices. This reduction in the cooling rate is responsible for a still ongoing debate as the cooling rate is widely believed to be an important factor for success of the vitrification protocols. Although several studies have shown that vitrification of oocytes (42), zygotes (11), blastocysts in closed carriers achieves good results in clinical studies $(3,7,9,26,43)$.

In a recent study, Chatzimeletiou et al. (44) investigate the effects of aseptic vitrification on the cytoskeleton and development of human blastocysts, by analysing survival rates and spindle and chromosome configurations by fluorescence and confocal laser scanning microscopy. Even though, 
there was a significantly higher incidence of abnormal spindles in the vitrified group compared with the fresh group, the high survival rate following warming and the large proportion of normal spindle/chromosome configurations suggests that aseptic vitrification at the blastocyst stage on day 5 does not adversely affect the development of human embryos and the ability of spindles to form and continue normal cell divisions.

There is no direct evidence of cryopreserved human oocytes/embryos becoming contaminated during cooling and cryostorage.

However, given the available information from bovine embryo studies and potential concerns with long term storage in open devices it would appear prudent to avoid such direct contact with $\mathrm{LN}_{2}$ during vitrification and subsequent storage by using closed devices.

\section{Step four: storage in $\mathrm{LN}_{2}$ containers, for how long?}

Since the goal of cryopreservation is to preserve cells and tissue in a solid state for long time periods, the stability of vitrified biological materials over time is an important issue and still matter of debate considering the thermodynamic aspect. The unstability phenomenom under $\mathrm{Tg}$ is of particular interest because the considerable molecular mobility that persists near and under $\mathrm{Tg}$ makes the stability of such storage temperatures non-obvious. In fact, crystalline solids are more stable because there is usually an energy of crystallization, that is released on crystallization with interactions between molecules. On the contrary, the conversion to a glass is not accompanied by exothermic heat of fusion but is accompanied by changes in the heat capacity of the sample (45). The glass transition can be understood on a molecular level as a loss of translational and rotational degrees of freedom over a particular measurement timescale, but leaving still bond vibration within a fixed molecular structure (46).

Although diffusion is practically non-existent below the glass transition temperature, small local movements of molecules related to relaxation have consequences for cryobiology (47). This is the consequence that amorphous solids usually happen when substances are cooled quickly and then move too slowly to orientate themselves.

It is commonly admitted in the field of thermodynamic cryobiology that once the glass is formed, it can be aged. Aged amorphous materials show de- creased physical and chemical reactivity compared to unaged materials.

Although several thousand children have been born after blastocyst vitrification, many aspects of this technique remain to be elucidated. New applications, such as fertility preservation, lead to long storage times of vitrified gametes or embryos but it remains to be determined if these vitrified embryos are stable over time. In the ART field, little is known about risks of prolonged storage of cryopreserved cells as vitrification is the solidification of a fluid without formation of crystalline structures - a physically disorganized unstable system. This raises the question if this state changes over time impairing survival and implantation potential of vitrified gametes and embryos. Subsequently, any potential impact on the health of the newborn is unknown.

The study of Wirleitner et al. (48) including the transfer of blastocysts which had been vitrified aseptically with the vitrisafe ${ }^{\circledR}$ device show that the storage of vitrified blastocysts in aseptic conditions neither impaired blastocyst viability (Survival rate after warming during the first year of storage was $83.0 \%$ compared with $83.1 \%$ after $5-6$ years of storage: NS) nor decreased pregnancy rates (clinical pregnancy rate after 1 year of storage was 40.0 versus $38.5 \%$ after 6 years: NS). In addition, no increase in the malformation rate over time was observed. In conclusion, we may advice that the strategy of "better safe than sorry" has no negative impact on the viability of vitrified blastocyst over a period of 6 years.

\section{Step five: is the warming process more important than the cooling one?}

Since the rate of cooling engendered a hot debate, it is surprising that little emphasis is put on the warming procedure. However, it has become obvious that the warming rate might play a more essential role in modulating survival after vitrification than the cooling rate. The primary cause of injury or cell death in a vitrification procedure is ice recrystallisation (devitrification) during warming and not failure to vitrify during cooling (49). A high warming rate prevents the vitreous water from re-crystallizing during the warming phase $(49,50)$.

It is well known that for any given concentration of $\mathrm{CP}$ the critical warming rate is much higher than the critical cooling rate (51). Consequently, the minimal concentration of $\mathrm{CP}$ to prevent crystal- 
lization during warming must be higher than during cooling. This means that it might be easier to maintain a vitrified state during the cooling than during the warming process for the same concentration of CP. If the warming rate is reduced by using devices isolating the drop containing the embryos higher intracellular concentrations of $\mathrm{CP}$ are needed in order to reduce the likelihood of re-crystallization. However these higher concentrations of CP might be toxic to the cells.

Hence, in order not to increase the concentration of $\mathrm{CP}$ above the toxic level, the biological material has to be warmed extremely rapidly. Concretely, most of open or closed devices used produce very high warming rates exceeding $20.000^{\circ} \mathrm{C} / \mathrm{min}$. Mechanistically, it has resulted in confounding of whether cooling rate or warming rate are important cooperative.

In conclusion, vitrification in aseptic conditions permits to achieve extremely high warming rate similar to the one obtained with open carrier device.

\section{Step six: dilution of the CP}

During warming water reenters the cells and $\mathrm{CP}$ are washed out. This has to be performed in a controlled way in order to avoid cellular damage. A too rapid influx of water is circumvented by a stepwise exposure to solutions containing reducing sucrose concentrations (30).

\section{Step seven: selection of warmed blasto- cysts before ET. Which one to select?}

Though some post-thaw morphological predictors have been investigated in slow freezing of blastocysts, e.g. immediate re-expansion $(52,53)$ or 24hour survival, no such data have yet been published for vitrified blastocysts. It has been suggested that as the result of the presence and size of the blastocoelic cavity, vitrified-warmed blastocysts experience several morphologic changes and become collapsed during cryopreservation. Thus, it is more difficult to score a vitrified blastocyst after warming than a fresh one (53).

Several factors (unrelated to vitrification method) are known to directly influence the fate of a cryopreserved blastocyst after thawing/warming and transfer. It is important to realize that survival rates in literature are hardly comparable due to the fact that some embryologists focus on immediate survival while others suggest an additional waiting period of 24 hours to facilitate control of growth (2). Differences in implantation rates may also be attributed to the fact that not all working groups apply assisted hatching to the thawed blastocysts (whilst shrunk), though this was found to improve outcome (2).

In detail, re-expansion of the blastocoele (and consequently the blastocyst) after thawing is expected within 24 hours after thawing $(2,52)$. However, immediate re-expansion, e.g. within the first two hours after warming, has not been used for prognostic purposes in vitrified blastocysts. Since in slow-freezing, approximately half of the frozen blastocysts turned out to re-expand immediately after 2-4 hours in culture (53) it is indicated that using vitrification a higher rate of re-expansion might be observed (54).

Even if it can be assumed that all viable blastocysts will re-expand after several more hours, any delay in this process could be the manifestation of altered osmotic and/or metabolic conditions (comparable to the situation found during blastocoele development when water enters the blastocoelic cavity via tight junctions either diffusing passively or being pumped actively).

To conclude, a fast re-expansion post warming is the best signal of blastocyst viability.

\section{Conclusions}

If we look back 15 years, a patient had to undergo several oocyte retrievals to achieve one pregnancy. As a result of our accomplishments regarding single blastocyst transfer in combination with the very efficient aseptic vitrification technique, we are now able to achieve higher cumulative pregnancy rates. From one oocyte retrieval, a woman may conceive several babies after transfer of one vitrified blastocysts at a time. It is now proven that aseptic vitrification with the closed vitrisafe ${ }^{\circledR}$ carrier is effective for blastocysts at different stage of development and quality.

Vitrification has to be adapt to the cooling/warming conditions. More intra and extracellular $\mathrm{CP}$ to keep the solution in a vitrification state is mandatory in case of a reduction in the cooling as well in the warming rate. However the fear about exposure of blastocyst to higher concentration of CPs is not justified.

Even though lower cooling rate, aseptic vitrification is an effective procedure if higher warming rates are achieved. Critical warming rates are typically two or more orders of magnitude greater than 
critical cooling rates. Finally the speed of re-expansion after warming is a good sign of viability.

\section{References}

1. Vanderzwalmen P, Bertin G, Debauche $\mathrm{CH}$, et al. Births after vitrification at morula and blastocyst stages: effect of artificial reduction of the blastocoelic cavity before vitrification. Human Reproduction. 2002;17:744-751.

2. Vanderzwalmen P, Bertin G, Debauche CH, et al. Vitrification of human blastocysts with the Hemi-Straw carrier: application of assisted hatching after thawing. Human Reproduction. 2003;18:1504-1511.

3. Vanderzwalmen P, Ectors F, Grobet L, et al. Aseptic vitrification of blastocysts from infertile patients, egg donors and after IVM. Reproductive BioMedicine Online. 2008;19:700-707.

4. Mukaida T, Nakamura S, Tomiyama T, et al. Vitrification of human blastocysts using cryoloops: clinical outcome of 223 cycles. Human Reproduction. 2003a;18:384-391.

5. Stehlik E, Stehlik J, Katayama KP, et al. Vitrification demonstrates significant improvement versus slow freezing of human blastocysts. Reproductive BioMedicine Online. 2005;11:53-57.

6. Liebermann J, Tucker MJ. Comparison of vitrification and conventional cryopreservation of day 5 and day 6 blastocysts during clinical application. Fertility and Sterility. 2006;86:20-26.

7. Stachecki J, Garrisi J, Sabino S, et al. A new safe, simple and successful vitrification method for bovine and human blastocysts. Reproductive BioMedicine Online. 2008; 17:360-367.

8. Van Landuyt L, Stoop D, Verheyen G, et al. Outcome of closed blastocyst vitrification in relation to blastocyst quality: evaluation of 759 warming cycles in a singleembryo transfer policy. Human Reproduction. 2011;26: 527-534.

9. Panagiotidis Y, Vanderzwalmen P, Prapas Y, et al. Open versus closed vitrification of blastocysts from an oocytedonation programme: a prospective randomized study. Reproductive BioMedicine Online. 2013;26:470-476.

10. Barnhart K. Are we ready to eliminate the transfer of fresh embryos in in vitro fertilization? Fertility and Sterility. 2014;102:1-2.

11. Vanderzwalmen P, Zech NH, Ectors F, et al. Blastocyst transfer after aseptic vitrification of zygotes: an approach to overcome an impaired uterine environment. Reproductive BioMedicine Online. 2012;25:591-599.

12. Shapiro B. Clinical rationale for cryopreservation of entire embryo cohorts in lieu of fresh transfer. Fertility and Sterility. 2014;102:3-9.

13. Ortega-Hrepich C, Stoop D, Guzmán 1, et al. A"freezeall" embryos strategy after in vitro maturation: a novel approach in women with polycystic ovary syndrome? Fertility and Sterility. 2013;100:1003-1007.

14. Weinerman R, Mainigi M. Why we should transfer frozen instead of fresh embryos: the translational rationale. Fertility and Sterility. 2014;102:10-18.

15. ALPHA Scientists in Reproductive Medicine, ESHRE Special Interest Group Embryology. Istanbul consensus workshop on embryo assessment: proceedings of an expert meeting. Reproductive BioMedicine Online. 2011;22:632-646.

16. Ebner T, Vanderzwalmen P, Shebl O, et al. Morphological Aspects of Human Blastocysts and the Impact of Vitrification. J Reproduktionsmed Endokrinol. 2011;8:13-20.

17. Ahlström A, Westin C, Reismer E, et al. Trophectoderm morphology: an important parameter for predicting live birth after single blastocyst transfer. Human Reproduction. 2011;26:3289-3296.

18. Honnma H, Baba T, Sasaki M, et al. Trophectoderm morphology significantly affects the rates of ongoing pregnancy and miscarriage in frozen-thawed single-blastocyst transfer cycle in vitro fertilization. Fertility and Sterility. 2012;98:361-367.

19. Ahlström A, Westin C, Wikland M, et al. Prediction of live birth in frozen-thawed single blastocyst transfer cycles by pre-freeze and post-thaw morphology. Human Reproduction. 2013;28:1199-1209.

20. Gardner DK, Schoolcraft WB. In vitro culture of human blastocysts In: Jansen R, Mortimer D (eds). Towards reproductive certainity: infertility and genetics beyond 1999. Parthenon Press, Carnforth, 1999, pp378-388.

21. Shaw-Jackson C, Bertrand E, Becker B, et al. Vitrification of blastocysts derived from fair to poor quality cleavage stage embryos can produce high pregnancy rates after warming. Journal of Assisted Reproduction and Genetics. 2013;30:1035-1042.

22. Mukaida T, Oka C, Goto T, et al. Artificial shrinkage of blastocoeles using either a micro-needle or a laser pulse prior to the cooling steps of vitrification improves survival rate and pregnancy outcome of vitrified human blastocysts. Human Reproduction. 2006;21:3246-3252.

23. Zech N, Lejeune B, Zech H, et al. Vitrification of hatching and hatched human blastocysts: effect of an opening in the zona pellucida before vitrifi cation. Reproductive BioMedicine Online. 2005;11:355-361.

24. Rall W, Fahy G. Ice-free cryopreservation of mouse embryos at -196 degrees C by vitrification. Nature. 1985; 313:573-575.

25. Leibo SP, Pool TB. The principal variables of cryopreservation: solutions, temperatures, and rate changes. Fertility and Sterility. 2011;96:269-276.

26. Kuwayama M, Vajta G, Leda S, et al. Comparison of open and closed methods for vitrification of human embryos and the elimination of potential contamination. Reproductive BioMedicine Online. 2005;11:608-614.

27. Leibo S. Water permeability and its activation energy of fertilized and unfertilized mouse ova. Journal of Membrane Biology. 1980;53:179-188.

28. Kasai M, Edashige K. Movement of water and cryoprotectants in mouse oocytes and embryos at different stages: relevance to cryopreservation. In: Ri-Cheng C, Quinn P (eds) Fertility Cryopreservation. Cambridge University Press, 2010:16-23.

29. Vanderzwalmen P, Ectors F, Grobet L, et al. Adaptation of a universal procedure for cryopreservation of different developmental stages: Is it conceivable? In: Varghese AC, Sjöblom P, Jayaprakasan K (eds) Practical Guide to Setting Up an IVF Lab, Embryo Culture Systems and Running the Unit. Jaypee Brothers Medical Publishers. 2013:2101-2110 and 118-131. 
30. Vanderzwalmen P, Connan D, Grobet L, et al. Lower intracellular concentration of cryoprotectants after vitrification than after slow freezing despite exposure to higher concentration of cryoprotectant solutions. Human Reproduction. 2013;28: 2101-2110.

31. Lane M, Schoolcraft WB, Gardner DK. Vitrification of mouse and human blastocysts using a novel cryoloop container-less technique. Fertility and Sterility. 1999; 72:1073-1078.

32. Mukaida T, Takahashi K, Kasai M. Blastocyst cryopreservation: ultrarapid vitrification using cryoloop technique. Reproductive BioMedicine Online. 2003b;6:221225.

33. Kuwayama M. Highly efficient vitrification for cryopreservation of human oocytes and embryos: The cryotop method. Theriogenology. 2007;67:73-80.

34. AbdelHafez F, Xu J, Goldberg J, et al. Vitrification in open and closed carriers at different cell stages: assessment of embryo survival, development, DNA integrity and stability during vapor phase storage for transport. BMC Biotechnology. 2011;11:29.

35. Bielanski A. A review of the risk of contamination of semen and embryos during cryopreservation and measures to limit cross-contamination during banking to prevent disease transmission in ET practices. Theriogenology. 2009;77:467-482.

36. Cobo A, Castello D, Weiss B, et al. Highest liquid nitrogen quality for vitrification process: micro bacteriological filtration of LN2. 16th World Congress on In Vitro Fertilization. 2011;Abstract P052: 289.

37. Parmegiani L, Accorsi A, Cognigni G.E, et al. Sterilization of liquid nitrogen with ultraviolet irradiation for safe vitrification of human oocytes or embryos. Fertility and Sterility. 2010;4:1525-1528.

38. Parmegiani L, Cognigni G, Bernardi S, et al. Efficiency of aseptic open vitrification and hermetical cryostorage of human oocytes. Reproductive BioMedicine Online. 2011;23:505-512.

39. Grout BW, Morris GJ. Contaminated liquid nitrogen vapour as a risk factor in pathogen transfer. Theriogenology. 2009;71:1079-1082.

40. Yan J, Suzuki J, Yu X.M, et al. Effects of duration of cryo-storage of mouse oocytes on cryo-survival, fertilization and embryonic development following vitrification. Journal of Assisted Reproduction and Genetics. 2011;28:643-649.

41. European parliament directive (EU Tissues and Cells Directive 2004/23/EC), URL: http://eurlex.europa.eu/Le-
xUriServ/site/fr/oj/2004/1_102/1_10220040407fr004800 58.pdf.

42. Papatheodorou A, Vanderzwalmen P, Panagiotidis Y, et al. Open versus closed oocyte vitrification system: a prospective randomized sibling-oocyte study. Reproductive BioMedicine Online. 2013;26:595-602.

43. Liebermann J. Vitrification of human blastocysts: an update. Reproductive BioMedicine Online. 2009;19(Suppl 4):4328.

44. Chatzimeletiou K, Morrison EE, Panagiotidis Y, et al. Cytoskeletal analysis of human blastocysts by confocal laser scanning microscopy following vitrification. Human Reproduction. 2012;27:106-113.

45. Baudot A, Boutron P. Glass-forming tendency and stability of aqueous solutions of diethylformamide and dimethylformamide, Cryobiology. 1998;37:187-199.

46. Morgana N, Spera F. Glass transition, structural relaxation, and theories of viscosity: a molecular dynamics study of amorphous CaAl2Si2O8. Geochimica et Cosmochimica Acta. 2001;65:4019-4041.

47. Wowk B. Thermodynamic aspects of vitrification. Cryobiology. 2010;60:11-22.

48. Wirleitner B, Vanderzwalmen P, Bach M, et al. The time aspect in storing vitrified blastocysts: its impact on survival rate, implantation potential and babies born. Human Reproduction. 2013;28:2950-2957.

49. Seki S, Mazur P. The dominance of warming rate over cooling rate in the survival of mouse oocytes subjected to a vitrification procedure. Cryobiology. 2009;59:75-82.

50. Seki S, Mazur P. Effect of warming rate on the survival of vitrified mouse oocytes and on the recrystallization of intracellular ice. Biology of Reproduction. 2008;79:727-737.

51. Fahy GM, Levy DI, Ali SE. Some emerging principles underlying the physical properties, biological actions, and utility of vitrification solutions. Cryobiology. 1987;24:196-213.

52. Van den Abbeel E, Camus M, Verheyen G, et al. Slow controlled-rate freezing of sequentially cultured human blastocysts: an evaluation of two freezing strategies. Human Reproduction. 2005;20:2929-2945.

53. Shu Y, Watt J, Gebhardt J, et al. The value of fast blastocyst re-expansion in the selection of a viable thawed blastocyst for transfer. Fertility and Sterility. 2008; 91:401-406.

54. Ebner T, Vanderzwalmen P, Shebl O, et al. Morphology of vitrified/warmed day-5 embryos predicts rates of implantation, pregnancy and live birth. Reprod Biomed Online. 2009;19:72-78. 